\title{
SIMPLIFICATION IN LIFE CYCLE ASSESSMENT OF SINGLE-FAMILY HOUSES: A REVIEW OF RECENT DEVELOPMENTS
}

\author{
Bernardette Soust-Verdaguer ${ }^{1}$, Carmen Llatas ${ }^{2}$, Antonio García-Martínez ${ }^{3}$
}

${ }^{1}$ Department of Building Construction I, Escuela Técnica Superior de Arquitectura, University of Seville, Reina Mercedes 2, 41012 Seville, Spain; email: $\underline{\text { bsoust@ }, \text { us.es }}$

${ }^{2}$ Department of Building Construction I, Escuela Técnica Superior de Arquitectura, University of Seville, Reina Mercedes 2, 41012 Seville, Spain; email: cllatas@us.es

${ }^{3}$ Department of Building Construction I, Escuela Técnica Superior de Arquitectura, University of Seville, Reina Mercedes 2, 41012 Seville, Spain; email: agarcia6@us.es

\section{Corresponding author:}

Bernardette Soust-Verdaguer

Mailing Address: Department of Building Construction I,

Escuela Técnica Superior de Arquitectura, University of Seville, Reina Mercedes 2, 41012 Seville, Spain. Phone: (+34) 954556591

Email: bsoust@,us.es 


\title{
SIMPLIFICATION IN LIFE CYCLE ASSESSMENT OF SINGLE-FAMILY HOUSES: A REVIEW OF RECENT DEVELOPMENTS
}

\begin{abstract}
Life Cycle Assessment (LCA) is globally recognized as one of the most complete methods for environmental assessment of buildings. Literature assumes that its applications in the building sector are prejudiced regarding complexity and difficulty. However, simplification is necessary, since it can facilitate LCA application in buildings. Moreover, growing interest on reducing environmental impact in the building sector, as well as the relevance of single-family houses on $\mathrm{CO}_{2}$ emissions have become key points on the wide spread of LCA. Therefore, this paper presents a research study about simplification in LCA recent studies applied to singlefamily houses. The review focuses on 20 cases that were analyzed according to ISO 14040, ISO 14044, EN 15978, and EN 15804 standards. The main objective was to identify the simplification strategies assumed in each paper, to clarify and to help to promote further developments on LCA. This paper examines system boundary definition, data sources, life cycle phases included, and environmental impact indicator calculated in case studies. Results show the variety of simplifications identified. They affect physical model definition, life cycle scenario definition and communication of results. In most cases, the functional unit was the complete building, the life cycle scenario definition included production, use and demolition phases, and the most considered environmental impact indicator was GWP. Finally, new challenges and recommendations were defined in order to establish common criteria to develop simplification strategies that allow results comparability in LCA of single-family houses.
\end{abstract}

Keywords: Life Cycle Assessment; simplified LCA; environmental assessment methods; environmental assessment impacts; review.

Abbreviations: AP, Acidification Potential; BIM, Building Information Modelling; CC, Climate Change; CED, Cumulative Energy Demand; EPD, Environmental Product Declaration; ET, Ecological Toxicity; GHG, Green House Gases; GW, Global Warming; HTP, Human Toxicity Potential; LCA, Life Cycle Assessment; LCI, Life Cycle Inventory; LCIA, Life Cycle Impact Assessment; MRR, Maintenance, Repair and Replacement; PMF, Particulate Matter Formation; WD, Water Depletion. 


\section{INTRODUCTION}

Current environmental problems have led to the development of measures for reducing the impact of human activities in the environment. These measures aim to control $\mathrm{CO}_{2}$ emissions, improve efficiency and save resource consumption. The last COP21 -also known as 2015 Paris Climate Conference- reinforced global compromises to reduce Global Warming and concluded by the signature of an agreement for reducing Carbon Emissions [1]. The building sector represents $19 \%$ of all global 2010 GHG emissions [2]; as well it is also a major consumer of natural sources [3]. Moreover, single-family houses have an important role in reducing environmental impacts of the building sector, since for example $60 \%$ of the $\mathrm{EU} \mathrm{CO}_{2}$ emissions of residential sector come from single-family houses [4].

LCA is recognized as a useful method to assess environmental impact in the building sector. Wiessenberger et al. [5] demonstrated the growing interest in LCA of building by the increasing number of publications of scientific studies in the last 20 years. Cabeza et al. [6] review, also demonstrated the use of LCA in the building sector through the organization of a large number of literature that includes construction products, systems, buildings, and civil engineering constructions. Lotteau et al. [7] developed a critical review of LCA applications at neighborhood level. The review exposes the trend towards the application of LCA on an urban scale, although literature about the subject is still scarce. The study demonstrates the heterogeneity of methodological choices and also develops recommendations to promote future research.

Buyle et al. [8] review focused on building applications of LCA and evidenced that LCA involves making a model that simplifies reality. Various LCA studies recognize that complexity and uncertainties are barriers for the widespread use of LCA of the built environment $[9,10])$. Moreover, "typical" applications of LCA in buildings are admitted as time-consuming and complex processes[11]. In the European Context several projects have been developed aiming to adapt LCA methods to the building sector. Moreover, the standardization work on LCA of buildings -CEN TC 350- defines EN 15804 [12] and EN 15978 [13] standards; and provides a methodological framework of the information to be included, stages and communication of results [14]. However, the advances identified on LCA of buildings, studies about simplification strategies focusing on single-family houses are still scarce.

It is assumed that application of the LCA method necessarily involves the simplification of reality; the challenge is the definition of a model so that simplifications do not considerably modify the final results [15]. Several studies [9-11,16-19] recognize the need of reliable simplifications in the application of LCA methods in 
buildings; especially to extend the scope to early stages of design, reduce effort in data acquisition, enable comparability of results, and allow results to be interpreted independently from the degree of specialization of the technicians. The key aspect is to know how simplifications can modify the representativeness of the results.

Therefore, this paper analyses recent developments in the application of LCA in single-family houses, focusing on methodological aspects and simplification strategies according to ISO 14040 [20], ISO 14044 [21], and EN 15978 [13] LCA standards. The main objective was to identify the simplifications and modelling assumed on each study, to clarify and to help promote further developments on LCA. Twenty selected cases are included in the following review. Some of them are complete building LCA application and others are focused on representatives systems, elements or parts of the building.

\section{LCA APPLIED TO BUILDINGS}

\subsection{Barriers on LCA applied to buildings}

The LCA method is based on the quantification of environmental impacts of a product throughout its life cycle, from "cradle to grave" [20,21]. In general terms, ISO 14040 standards establish the stages for LCA application. LCA approach adapted to building is defined in EN 15978 [13] that represents a methodological guide for the quantification of environmental impacts on buildings.

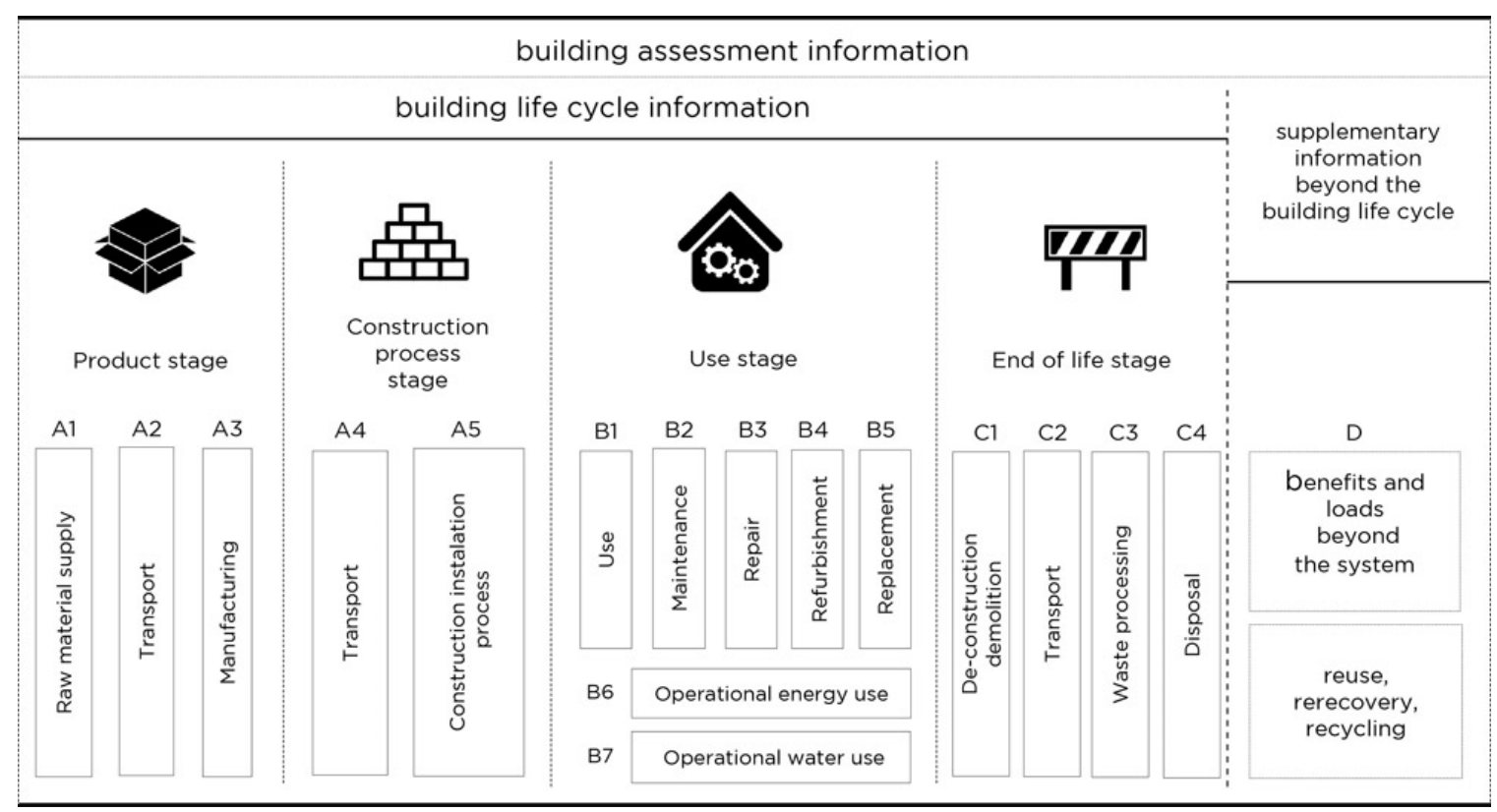

Fig. 1. Scheme of the information modules for the different stages of evaluation of the building based on 15978 [13]. 
EN 15978 [13] is organized according to the "modules principle" of building life cycle, including the four stages of life cycle - product, construction, use, end of life- ([13,19]. These stages and modules are shown in Figure 1 and cover from A1 to C4 "impacts and environmental aspects" developed within the system boundary, while D modules cover benefits and loads that go beyond the system boundary.

The standards also provide the guidelines for functional unit, system boundary and scenario definitions. The functional unit is a "quantified performance of a product system for use as a reference unit" [21], and it includes the physical and functional characteristics of the building. System boundary limits the processes included in the assessment. The scenarios are hypothesis applied to the subject of study that relates physical characteristics with time variable [13]. EN 15978 [13] also defines the structure of results communications and a list of environmental indicators that have to be considered as shown in Table 1. Results have to be expressed according to the list of indicators included in Table 1, separated by stage (production, construction, use, end of life, recycle) and by modules (A1-D) [13].

Table 1. List of indicators described in EN 15978 [13] standard.

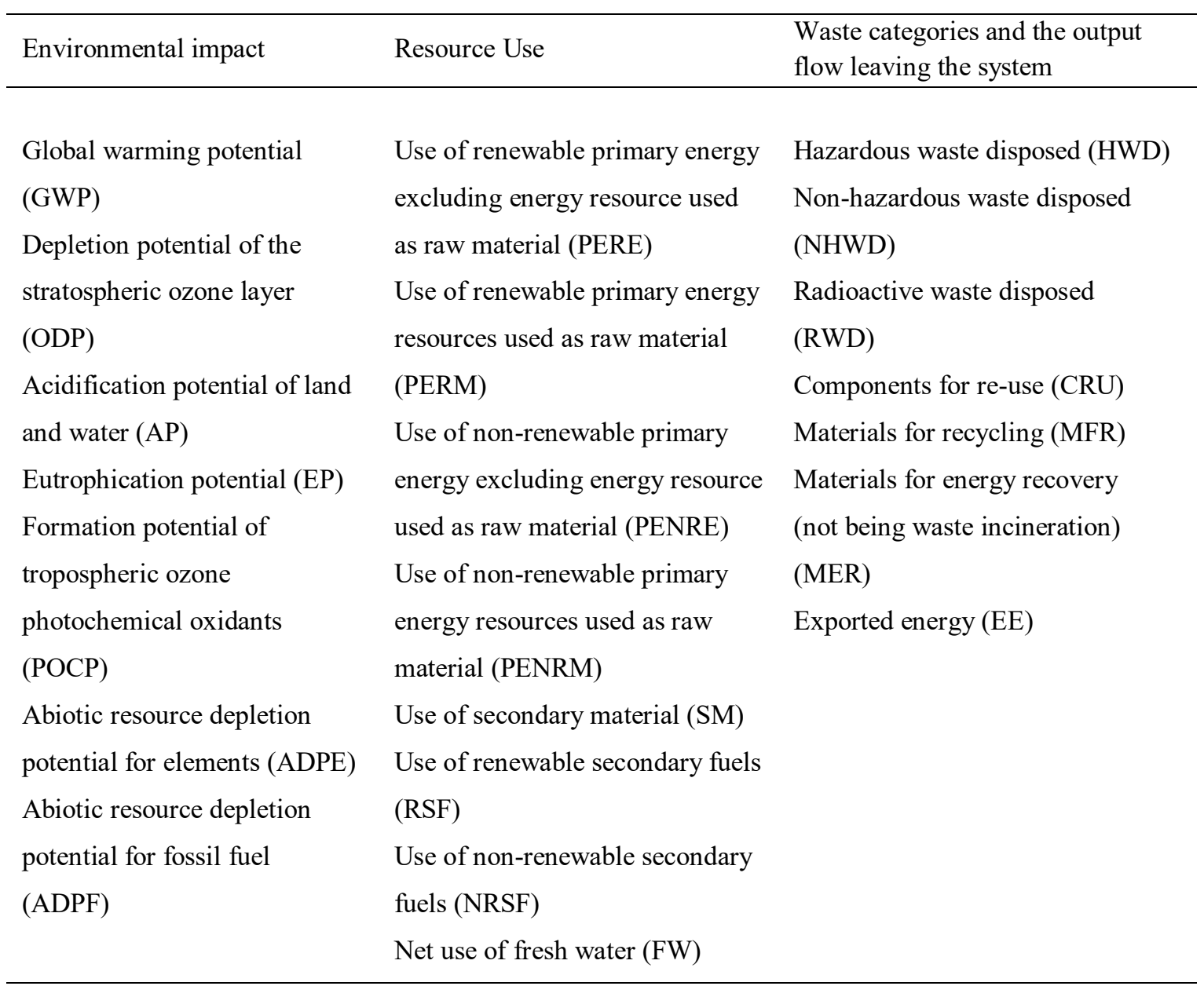


EN 15804 [12] provides calculation rules for Environmental Product Declarations (EPD) and it also includes a methodological framework of Modules A1-A3 of EN 15978 standard [13]. Otherwise, the EPD of building products can be used as product stage modules in EN 15978 [13]. Passer et al. [22] shown the growing interest in EPD of building products, evidenced by the high number of EPD programs that guide CEN/TC 350 standards "Sustainability of construction works".

In the European context the application of LCA method in building sector is promoted by several projects as: REGENER [23], Annex 31 IEA [24], PRESCO [25], IMPRO-Building [26], ENSLIC Building [27], LoReLCA [28] and EeB Guide Project [14]. The EeB Guide Project [14] defines three types of LCA in buildings: Screening, Simplified and Complete. The classification is developed according to system boundary definition, the experience of the practitioner, data availability and the state of development of the product or building being assessed [14].

EeB Guide Project [14] also establishes a life cycle stages definition for each LCA study types. For Complete LCA study, all stages and modules defined in EN 15978 [13] are compulsory. In Screening and Simplified LCA types, several stages and modules are optional; depending on the relevance and data availability. For Screening LCA only the following modules are compulsory: Product stages for the building envelope, structure and foundation (A1-3), Operational use of energy (B6) and water (B7). For Simplified LCA type, in addition to compulsory modules considered in Screening type (A1, A2, A3, B6 and B7), the use and end of life stages are also partially included; modules as Replacement (B4), Waste treatment (C3) Disposal (C4) and Benefits (D) are even considered compulsory [14].

Despite the fact that LCA is the most complete tool for the environmental assessment of buildings, their application in buildings is not yet widespread. Some of the main difficulties are the extensive and exhaustive amount of information required, as well as the required experience of the practitioner for calculating impacts [17]. Moreover, architects and technicians involved in the early stages of the design have prejudices about the complexity, precision and arbitrary results [17].

Another difficulty in LCA application is the existence of different methods for impact calculation, so depending on the method different results for identical cases [8] can be obtained. Each method has differences in weighting impact categories; this can lead to different measures for reducing environmental burdens [8]. 


\subsection{Simplifications in $L C A$}

In the Spanish context, 150041-1998EX UNE [29] establishes general criteria to simplify the LCA method. The regulation establishes the simplifications that can be carried out in the life cycle inventory phase and the life cycle impact assessment phase. It means that the life cycle inventory analysis can be reduced to the main elements and processes, and the impact assessment phase can be simplified to a few impact categories mandatory and optional- [29]. Zabalza et al. [17] assessed a residential building in Spain by developing a simplified LCA method. The study focuses on the calculation of operational energy consumption and $\mathrm{CO}_{2}$ emissions

Relevant research papers about LCA of residential buildings and building materials have also simplified the application of LCA. Malmqvist et al. [9] recommend for simplifying the LCA method of buildings: (1) reducing the data acquisition phase -e.g. focusing on larger building elements-, (2) simplifying inventory analysis -e.g. focusing on the most important substances that contribute to a certain impact category-; (3) simplifying the calculation by focusing on a few impact categories and finally, (4) reducing the time of data applications by using CAD applications

Time-reduction of data acquisition strategies are developed in Basbagill et al. [11]. In this study, the uses of Building Information Modelling (BIM) applications to quantify building materials were developed to assess a residential building in USA. The study proposes a method that integrates BIM, LCA, energy simulation, Maintenance, Repair and Replacement (MRR) schedule, and sensitivity analysis software. The method can quickly integrate LCA in the early stages of design.

Moreover, Karami et al. [30] simplified data acquisition in the production phase by using EPDs of different building envelopes. The study compares the environmental impact of building envelopes of three residential buildings located in Sweden. They also reduce LCA stages by including only production of envelope materials and energy consumption of the building in operational phase. Modules A4-A5, B1-B5 B7, C and D are included in the study.

Kellenberger and Althaus [16] aimed to define the relevance of materials and processes on occasions not considered in simplified LCA of building components. Results demonstrate the incidence of transport and ancillary materials in the impact indicators, and also evidence the irrelevance of building processes and waste cutting. 
On the other hand, Takano et al. [19] studied the application of the standards EN 15978 [13] and EN 15804 [12] to a referenced wooden building. The authors demonstrate that the standards provide a framework for building assessments, though several difficulties are identified. Data complexity and uncertainties in data collection are underlined. Simplifications in construction phase assessments are suggested, as well as changes in the communication of results. They propose a communication system based on a common method that stimulates environmental consciousness in society.

Therefore, despite literature assumes the need to introduce simplifications in the LCA method; few studies have studied the incidence of simplification strategies in the most significant typology in terms of $\mathrm{CO}_{2}$ emissions.

\section{MATERIALS AND METHODS}

The method consisted of two stages: 1) selection of case studies that refer to literature search and the criteria of the cases studies selected, 2) analysis of the LCA applied to single-family houses that includes the LCA phases analysis and the life cycle stages.

\subsection{Criteria for selection of case studies}

Over the last five years, after the publication of EN 15978 [13] and 15804 [12] standards, several studies have been published about LCA of buildings. This paper carried out a selection of recent publications on LCA in single-family houses; typology responsible for the majority of $\mathrm{CO} 2$ emissions of building sector. The criteria selected were: 1) publications covered by Scopus and included in the Journal Citation Report, 2) studies based on LCA applied to single-family houses, and 3) studies conducted in the last five years. Selected papers were applications of the LCA method, in which simplifications were identified and analyzed according to LCA buildings standards.

\subsection{Criteria for analysis of the LCA applied to single-family houses}

The analysis was organized in two parts, the first one referred to LCA key phases and the second one was based on life cycle stages definition. The criteria to organize case study was centered on the classification into European and Non-European cases, the scope of the LCA application-partial or complete-, and the characteristics of the house -typical or energy efficient certificated-. 


\subsubsection{Criteria for analysis of LCA phases}

References to simplification strategies for LCA focus attention on methodological aspects. Therefore, the analysis of simplification strategies in the case studies was centered on functional units, data sources and environmental impact indicators. The goal and scope definition analysis -included in Table 2- was analyzed according to the following aspects: 1) the reference standard to develop LCA; 2) the object of study and a brief characteristic of it -typical or energy efficient certificated-; 3) the functional unit definition -partial and complete LCA applications-, 4) the service life time considered. Table 3 was centered on the data sources and information structure. It analyzes data sources to obtain the main information modules: 1) product -including raw material, transport and manufacturing-; 2) construction, 3) use -including use, maintenance, replacement, repair, refurbishment-, 4) end of life -including demolition, waste processing and disposal-, 5) recycle, 6) transport to construction site, 7) transport to landfill site, 8) energy consumption and 9) water consumption. Table 4 consisted on the analysis of the results including: 1) calculation tools; 2) impact calculation method; 3) environmental impact indicators.

\subsubsection{Criteria for analysis the life cycle stage}

From the literature review, it was detected that LCA of buildings can be focus on certain life cycle stages. Several cases $[17,30]$, for example, were focused on product stages as a strategy to reduce the amount of data and complexity on LCA application. Based on that fact, LCA stages definition became a relevant aspect on LCA of single-family houses. In order to analyze this aspect, a grid was organized to identify stages and modules described in EN15978 [13] -European reference in LCA of buildings. Nevertheless, several non-European cases [31-34] were included. Table 5 resumes the information modules covered by the LCA application. It was focused on identifying the stages that have been selected, as well as the most relevant in case studies.

\section{RESULTS}

\subsection{Selected case studies}

An overview of recent developments of LCA applied to single-family houses is shown in Table 2, 3, 4 and 5. It includes 14 European cases, 5 Non- European cases and a case that compares both situations.

\subsection{Analysis of LCA phases}

\subsubsection{Goal and scope definition phase}

The definition of the functional unit had a key role in LCA of single-family houses. According to Cabeza et al. [6], LCA of buildings can be classified into product application (EDP) and building application. The papers 
referred to building application, but in several cases the complete building was considered as the functional unit and in other cases the functional unit was a part of the building placed in the building e.g. envelope, windows,

roof, shading, etc. Most of the papers have considered the complete building as a functional unit. Passivehaus standards and energy efficient cases were analyzed; some cases were compared to standard houses. Fouquet et al (2015) develop a LCA of a "Passivehaus standard" single-family houses located in France. The study compares the same house built with different materials and techniques: Timber frame (landfill and incineration), Cast concrete, Concrete blocks cavity walls. Mosteiro-Romero et al. [35] compare a four-bedroom two-story singlefamily house in Chur, Switzerland, certified Minergie-P building design, with an existing four-bedroom twostory single-family house in Monmouth County (New Jersey) LEED-homes certified. Lewandowska et al. [36] described a LCA of four single-family houses: a traditional masonry building, a passive masonry building, a traditional wooden building, a passive wooden building. The study compared different scenarios on energy consumption: Baseline scenario, Scenario PL 2020, Scenario PL 2025, Scenario PL 2030 and Scenario PL 2050.

In addition, typical houses were assessed. Cuéllar-Franca \& Azapagic [37] for instance study the environmental impacts of the housing sector in UK by application of the LCA method in the most common housing typologies. They assume a complete building LCA application and consider product, construction, use and end of life stages, benefits / loads are partially considered. Rossi et al. [38] compare the same house located in three different towns: Brussels (Belgium), Luleå (Sweden) and Coimbra (Portugal). Iddon et al. [39] compare different construction scenarios for a single-family house typology located in UK. Takano et al. [40] develop a LCA to a hypothetical building model and define as functional unit the space surrounded by exterior wall, roof and floor. Houlihan et al. (2014) consider as functional unit a typical timber single-family house, modelled in BIM. Oyarzo \& Peuportier [33] develop a model based on LCA, adapted to a Chilean context. The model is used to assess three alternatives of typical houses located in different climate zone: Punta Arenas, Santiago de Chile, Antofagasta and Puerto Montt.

Although a complete building has been considered as the functional unit, several cases do not include interior furniture and installations. Mosteiro-Romero et al. [35] for example does not include: furniture, lighting fixtures and appliances, site work outside the building footprint, landscaping and utilities outside the building.

Moreover, the functional unit could be considered as a representative part of the building, e.g. $1 \mathrm{~m}^{2}$ of floor area. Dahlstrøm et al. [41] for example, evaluate different ways of reducing the environmental impact of 
Table 2. Definition and scope of reviewed papers.

\begin{tabular}{|c|c|c|c|c|c|c|}
\hline & Reference & Location & $\begin{array}{l}\text { Reference } \\
\text { Standard }\end{array}$ & Scope & Functional unit & $\begin{array}{l}\text { Service } \\
\text { lifetime } \\
\text { (years) }\end{array}$ \\
\hline \multirow[t]{14}{*}{$\begin{array}{l}\text { 1. European } \\
\text { Cases }\end{array}$} & Houlihan et al., 2014 & Norway & EN 15978 & $\begin{array}{l}\text { Typical Single-family } \\
\text { house }\end{array}$ & $1 \mathrm{~m} 2$ of useful area & 60 \\
\hline & Gervasio et al., 2014 & Portugal & $\begin{array}{l}\text { EN } 15978 / \\
\text { EN } 15643\end{array}$ & Single family-house & $\begin{array}{l}1 \mathrm{~m} 2 \text { useful area of } \\
\text { Macro-components }\end{array}$ & 50 \\
\hline & Dahlstrøm et al., 2012 & Norway & NA & $\begin{array}{l}\text { Two Single family- } \\
\text { house (conventional } \\
\text { and Passivhaus }\end{array}$ & $1 \mathrm{~m} 2$ useful area & 50 \\
\hline & Proietti et al., 2013 & Italy & $\begin{array}{l}\text { ISO } 14040 \\
/ 14044\end{array}$ & $\begin{array}{l}\text { Single-family house } \\
\text { (Passivhaus standard) }\end{array}$ & $1 \mathrm{~m} 2$ of useful area & 70 \\
\hline & Motuzienè et al., 2016 & Lithuania & NA & $\begin{array}{l}\text { Energy efficient } \\
\text { Single-family house }\end{array}$ & $\begin{array}{l}1 \mathrm{~m} 2 \text { of heated floor } \\
\text { area }\end{array}$ & 100 \\
\hline & Takano et al., 2015 & Finland & EN 15978 & $\begin{array}{l}\text { Hypothetical building } \\
\text { model }\end{array}$ & Net heated floor area & 50 \\
\hline & $\begin{array}{l}\text { Cuéllar-Franca \& } \\
\text { Azapagic, 2012Gervasio }\end{array}$ & UK & $\begin{array}{l}\text { ISO } 14040 / \\
14040\end{array}$ & $\begin{array}{l}\text { Three typical } \\
\text { Single family-house }\end{array}$ & Complete Building & 50 \\
\hline & Iddon et al., 2013 & UK & ISO 14040 & Single-family house & Complete Building & 60 \\
\hline & Monteiro \& Freire., 2012 & Portugal & $\begin{array}{l}\text { ISO } 14040 / \\
14044\end{array}$ & Single-family house & Complete Building & 50 \\
\hline & Rosselló-Batle et al., 2015 & Spain & NA & Single-family house & Complete Building & 50 \\
\hline & Rossi et al., 2012 & $\begin{array}{l}\text { Brussels Luleå } \\
\text { and Coimbra }\end{array}$ & NA & Three houses & Complete Building & 50 \\
\hline & Lewandowska et al., 2013 & Poland & $\begin{array}{l}\text { ISO } 14040 / \\
14044\end{array}$ & $\begin{array}{l}\text { Four single-family } \\
\text { house }\end{array}$ & Complete Building & 100 \\
\hline & Fouquet et al., 2015 & France & EN 15978 & $\begin{array}{l}\text { Three single family- } \\
\text { house } \\
\text { (Passivhaus standard) }\end{array}$ & Complete Building & 100 \\
\hline & Peuportier et al., 2013 & France & NA & $\begin{array}{l}\text { Single-family house } \\
\text { (Passivhaus standard) }\end{array}$ & Complete Buildings & $50 / 100$ \\
\hline \multirow[t]{5}{*}{$\begin{array}{l}\text { 2. Non- } \\
\text { European } \\
\text { cases }\end{array}$} & Babaizadeh et al., 2015 & US & $\begin{array}{l}\text { ISO } 14040 \\
\text { ASTM standard } \\
\text { (for MADA) }\end{array}$ & $\begin{array}{l}\text { Typical Single } \\
\text { family-house }\end{array}$ & Shading for windows & 40 \\
\hline & Utama et al., 2012 & Indonesia & ISO 14040 & $\begin{array}{l}\text { Typical stand-alone } \\
\text { residential house }\end{array}$ & Building envelope & 40 \\
\hline & Hanandeh, 2015 & Jordan & ISO 14040 & $\begin{array}{l}\text { Six single family- } \\
\text { house }\end{array}$ & Complete Building & 50 \\
\hline & Islam et al., 2014 & Australia & ISO 14044 & $\begin{array}{l}\text { Typical Australian } \\
\text { house }\end{array}$ & Complete Building & 50 \\
\hline & $\begin{array}{l}\text { Oyarzo \& Peuportier, } \\
2014\end{array}$ & Chile & ISO 14040 & Single-family house & Complete Building & $30 / 50 / 80 / 100$ \\
\hline $\begin{array}{l}3 . \\
\text { European and } \\
\text { Non-European } \\
\text { cases }\end{array}$ & $\begin{array}{l}\text { Mosteiro-Romero et al., } \\
2014\end{array}$ & $\begin{array}{l}\text { US / } \\
\text { Switzerland }\end{array}$ & $\begin{array}{l}\text { ISO } 14040 / \\
14044\end{array}$ & $\begin{array}{l}\text { Two Single-family } \\
\text { house }\end{array}$ & Complete Building & 65 \\
\hline
\end{tabular}


building life cycle by considering two types of single-family houses -a conventional and a passive house standard- in Norway. Moreover, the study compares a renewable heating system with a Standard Norwegian system based on electricity. The functional unit is $1 \mathrm{~m}^{2}$ of useable floor area of a wooden house with four inhabitants.

Others studies were focused on assessing the environmental impact of an element or system that compose the building. Babaizadeh et al. [31] develop a LCA of 5 types of shading windows located in different climate zones of US. The study shows the environmental impact of the shading material tested on a typical single-family house. Hanandeh [32] applies the LCA method to evaluate the efficiency of different exterior wall construction of typical single -family houses. Gervasio et al. [42] develop an LCA method for quantifying cycle embodied impacts and energy consumption at early stages of design. The study focuses on a macro-components approach that reduces the amount of information and complexity on the application of the LCA method.

The use of standards to develop the LCA application was confirmed in $80 \%$ percent of cases studies. Almost $30 \%$ of European cases have developed LCA applications according to EN 15798 [13]. Non-European cases and $30 \%$ of European cases have been based on ISO standards [20].

\subsubsection{Life Cycle Inventory (LCI) phase}

According to literature, the Life Cycle Inventory (LCI) aims to quantify input and output flows and it is considered the most complex and time-consuming process [11]. Among the analysis of the selected papers a wide variety of data sources were identified. Results described in Table 3 were organized conforming to EN 15978 [13] information structure, as follow: building model -including materials, components and systems that compose the building-; building process - including transport, maintenance, repair, replacements, refurbishments, demolition, waste processing, recycling-; operational consumption -including energy and water consumption-.

The Building Model is a physical reference of the subject of study and it includes all the products and components that composed the building. For the quantification and organization of data certain simplifications strategies were identified. The use of BIM to quantify building material was identified in Iddon, et al. [39] and in Houlihan et al. [43]. On the other hand, the use of other data structures to organize information about building materials and products was detected in Islam et al. [44] Gervasio et al. [42], Dahlstrøm et al. [41]. 
Building process refers to processes and activities related to the life cycle of the building including: transport, construction, maintenance, repair, refurbishment, replacement, demolition, and disposal. These processes were grouped as: Construction, Use, Demolition, Recycle, Transport to construction site and to landfill as shown Table 2. In the Building process major uncertainties were identified, because of the need to define processes adapted to case studies. Table 3 evidenced that most of the case studies develop estimations for building processes in use and demolition stage.

Most of the case studies use Ecoinvent, a generic database for obtaining environmental data about construction materials. However, Proietti et al. [45] and [46] Cuéllar-Franca \& Azapagic did not specify the Ecoinvent version applied in the LCA. The Ecoinvent database version 2.2 was used in $15 \%$ of the case studies $[35,36,43]$. Ecoinvent databases represents one of the most complete and widely used databases as other authors indicated $[47,48]$. Although it is recognized that the use of generic databases can help and reduce significantly the amount of data and complexity during product phase, representativeness of data cannot be assured [14].

On the other hand, several cases that regional LCI have not been available, data used from generic databases has been adapted to reflect regional characteristics. Islam et al. [44] for instance, use wherever possible Australian region specific database (AusLCI) and data from the European Ecoinvent database "adjusted" to Australian electricity and transportation. Cuéllar-Franca \& Azapagic [37] carry out data collection by using Ecoinvent and GaBi V4.3. It has also used other literature sources as: Berge [49], Anderson et al. [50], DEFRA [51], Dewulf et al. [52], Prime et al. [53], Ortiz et al. [54], Utley \& Shorrock [55], Zabalza et al. [17]. However, the mix of different databases has to be carefully carried out. In that sense, Takano et al. [48] compare five databases used in buildings LCA -Gabi 6, Ecoinvent v3.0, IBO, CFP, and Synergia-. The study demonstrates that in spite of the fact that the compared database shows similar trends in assessment results, there are numerical differences between the studied databases.

Other cases were based on local data sources. For instance, Hanandeh [32] estimates transport based on distances from factory to construction site. Dahlstrøm et al. [41] develop data collection about construction based on calculations and contact with external companies as DeWalt Power Tools [56], SINTEF Byggforsk [57], Horn [58], F-Tech [59].

Operational consumption is related to energy consumption and water use of the building. In most cases, it was detected that energy consumption was calculated by dynamic thermal simulations tools that were in several cases adapted to regional energy efficiency regulations. For instance, Rosselló-Batle et al. [60] use LIDER- 
CALENER -a Spanish tool-, Peuportier et al, [61] and Fouquet et al. [62] employ PLEIADES+Comfie -a French tool-, Houlihan et al. [43] use SIMIEN -Norwegian tool-.

During the modelling of energy consumption it was detected that several cases do not include internal equipment from activities developed in the building. Monteiro \& Freire [63] for example, do not include in energy consumption: electric appliances, lighting, cooking, and domestic hot water. Despite the fact that it restricted the comparability of results, the strategy could reduce the complexity of data sources by focusing the attention on the envelope and the thermal performance of the building. 
Table 3. Data sources include on reviewed papers.

\begin{tabular}{|c|c|c|c|c|c|c|c|c|c|c|c|}
\hline & & \multicolumn{2}{|c|}{ Building model } & \multicolumn{6}{|c|}{ Building process } & \multicolumn{2}{|c|}{ Operational consumption } \\
\hline \multicolumn{2}{|c|}{ Reference } & $\begin{array}{l}\text { Material and } \\
\text { products } \\
\text { quantification }\end{array}$ & $\begin{array}{l}\text { Product stage } \\
(\mathbf{A 1}, \mathbf{A 2}, \mathbf{A 3})\end{array}$ & $\begin{array}{l}\text { Construction } \\
\text { stage } \\
\text { (A5) }\end{array}$ & $\begin{array}{l}\text { Use stage } \\
\text { (B1, B2, B3, B4, } \\
\text { B5) }\end{array}$ & $\begin{array}{l}\text { End of Life } \\
\text { stage } \\
(\mathrm{C} 1, \mathrm{C} 3, \mathrm{C} 4)\end{array}$ & $\begin{array}{l}\text { Recycle stage } \\
\text { (D) }\end{array}$ & $\begin{array}{l}\text { Transport to } \\
\text { construction } \\
\text { site (A4) }\end{array}$ & $\begin{array}{l}\text { Trasnsport } \\
\text { to lanfill site } \\
\text { (C2) }\end{array}$ & $\begin{array}{l}\text { Energy } \\
\text { Consumption } \\
\text { Calculation } \\
\text { (B6) }\end{array}$ & $\begin{array}{l}\text { Water } \\
\text { Consumption } \\
\text { (B7) }\end{array}$ \\
\hline \multirow[t]{9}{*}{$\begin{array}{l}\text { 1. European } \\
\text { cases }\end{array}$} & $\begin{array}{l}\text { Houlihan et al., } \\
2014\end{array}$ & BIM model & Ecoinvent v2.2 & - & - & - & - & - & - & $\begin{array}{l}\text { Norwegian } \\
\text { official standards } \\
\text { [64-67] }\end{array}$ & - \\
\hline & $\begin{array}{l}\text { Gervásio et al., } \\
2014\end{array}$ & $\begin{array}{l}\text { Uniformat } \\
\text { classification } \\
\text { scheme }\end{array}$ & GaBi 6 & - & - & - & - & - & - & Design Builder & - \\
\hline & $\begin{array}{l}\text { Dahlstrøm et al., } \\
2012\end{array}$ & $\begin{array}{l}\text { Previous } \\
\text { research } \\
{[68]}\end{array}$ & Ecoinvent v2.0 & $\begin{array}{l}\text { Previous research } \\
\text { [56-59] }\end{array}$ & - & - & - & - & - & $\begin{array}{l}\text { Authors } \\
\text { estimations }\end{array}$ & $\begin{array}{l}\text { Statistic } \\
\text { estimations } \\
\text { [69] }\end{array}$ \\
\hline & $\begin{array}{l}\text { Motuzienè et al., } \\
2016\end{array}$ & BIM model & SimaPro libraries & - & - & - & - & - & - & DesignBuilder & - \\
\hline & $\begin{array}{l}\text { Proietti et al., } \\
2013\end{array}$ & & $\begin{array}{l}\text { Ecoinvent and } \\
\text { Previous research } \\
{[70-72]}\end{array}$ & $\begin{array}{l}\text { Authors } \\
\text { estimations and } \\
\text { Previous research } \\
{[16,70-78]}\end{array}$ & $\begin{array}{l}\text { Authors } \\
\text { estimations and } \\
\text { Previous research } \\
{[16,70-75,77-81]}\end{array}$ & $\begin{array}{l}\text { Authors } \\
\text { estimations and } \\
\text { Previous research } \\
{[16,70-} \\
72,74,75,77,79,80 \\
, 82-85]\end{array}$ & $\begin{array}{l}\text { Authors estimations } \\
\text { and previous } \\
\text { research }\end{array}$ & $\begin{array}{l}\text { Cooperation } \\
\text { with the } \\
\text { designer/owner, } \\
\text { technical data }\end{array}$ & - & PHPP & - \\
\hline & $\begin{array}{l}\text { Takano et al., } \\
\text { 2015a }\end{array}$ & - & Ecoinvent v3.01 & - & - & $\begin{array}{l}\text { Finnish Ministry } \\
\text { of the } \\
\text { Environment }\end{array}$ & $\begin{array}{l}\text { EQUA Simulation } \\
\mathrm{AB}\end{array}$ & $\begin{array}{l}\text { EQUA Simulation } \\
\mathrm{AB}\end{array}$ & - & $\begin{array}{l}\text { EQUA Simulation } \\
\mathrm{AB} \\
\text { and other sources } \\
{[86]}\end{array}$ & - \\
\hline & $\begin{array}{l}\text { Cuéllar-Franca \& } \\
\text { Azapagic, } 2012\end{array}$ & $\begin{array}{l}\text { Previous } \\
\text { research } \\
{[50,87,88]}\end{array}$ & $\begin{array}{l}\text { Ecoinvent, } \\
\text { GaBi V4.3 } \\
\text { and previous } \\
\text { research [49-55] }\end{array}$ & $\begin{array}{l}\text { Previous research } \\
\text { [73] }\end{array}$ & $\begin{array}{l}\text { Previous research } \\
\text { [50] }\end{array}$ & $\begin{array}{l}\text { Authors } \\
\text { estimations }\end{array}$ & Authors estimations & $\begin{array}{l}\text { Previous research } \\
{[54]}\end{array}$ & $\begin{array}{l}\text { Authors } \\
\text { estimations }\end{array}$ & $\begin{array}{l}\text { Previous research } \\
{[55,89]}\end{array}$ & $\begin{array}{l}\text { Previous } \\
\text { research } \\
{[55]}\end{array}$ \\
\hline & Iddon et al., 2013 & BIM model & ICE database & - & - & - & - & - & - & $\begin{array}{l}\text { ISO standard [90], } \\
\text { BRE Domestic } \\
\text { Energy Model, } \\
\text { and other sources } \\
{[91]}\end{array}$ & - \\
\hline & $\begin{array}{l}\text { Monteiro \& } \\
\text { Freire, } 2012\end{array}$ & - & $\begin{array}{l}\text { Sources based on } \\
\text { Ecoinvent v2.0 } \\
\text { [92-94] }\end{array}$ & $\begin{array}{l}\text { Authors } \\
\text { estimations and } \\
\text { Previous research } \\
{[16]}\end{array}$ & $\begin{array}{l}\text { Authors } \\
\text { estimations }\end{array}$ & - & - & $\begin{array}{l}\text { Authors } \\
\text { estimations }\end{array}$ & - & $\begin{array}{l}\text { RCCTE } \\
\text { calculation } \\
\text { method }\end{array}$ & - \\
\hline
\end{tabular}


Table 3. (continued)

\begin{tabular}{|c|c|c|c|c|c|c|c|c|c|c|c|}
\hline \multicolumn{4}{|c|}{ Building model } & \multicolumn{6}{|c|}{ Building process } & \multicolumn{2}{|c|}{ Operational consumption } \\
\hline & Reference & $\begin{array}{l}\text { Material and } \\
\text { products } \\
\text { quantification }\end{array}$ & $\begin{array}{l}\text { Product stage } \\
(\mathbf{A 1}, \mathbf{A 2}, \mathbf{A 3})\end{array}$ & $\begin{array}{l}\text { Construction } \\
\text { stage } \\
\text { (A5) }\end{array}$ & $\begin{array}{l}\text { Use stage } \\
\text { (B1, B2, B3, B4, } \\
\text { B5) }\end{array}$ & $\begin{array}{l}\text { End of Life } \\
\text { stage } \\
(\mathrm{C} 1, \mathrm{C} 3, \mathrm{C} 4)\end{array}$ & $\begin{array}{l}\text { Recycle stage } \\
\text { (D) }\end{array}$ & $\begin{array}{l}\text { Transport to } \\
\text { construction } \\
\text { site (A4) }\end{array}$ & $\begin{array}{l}\text { Trasnsport } \\
\text { to lanfill site } \\
\text { (C2) }\end{array}$ & $\begin{array}{l}\text { Energy } \\
\text { Consumption } \\
\text { Calculation (B6) } \\
\end{array}$ & $\begin{array}{l}\text { Water } \\
\text { Consumption } \\
\text { (B7) }\end{array}$ \\
\hline \multirow{5}{*}{$\begin{array}{l}\text { 1. European } \\
\text { cases } \\
\text { (cont.) }\end{array}$} & $\begin{array}{l}\text { Rosselló-Batle et al., } \\
2015\end{array}$ & - & BEDEC & - & - & - & - & - & - & $\begin{array}{l}\text { LIDER }+ \\
\text { CALENER }\end{array}$ & - \\
\hline & Rossi et al., 2012 & - & $\begin{array}{l}\text { Bees } \\
\text { WORLDSTEEL, } \\
\text { CRTI }\end{array}$ & - & - & - & - & - & - & Pleaides + Comfie & - \\
\hline & $\begin{array}{l}\text { Lewandowska et al., } \\
2013\end{array}$ & - & Ecoinvent v2.2. & - & $\begin{array}{l}\text { Authors } \\
\text { estimations }\end{array}$ & - & - & $\begin{array}{l}\text { Vehicle route } \\
\text { planning software }\end{array}$ & $\begin{array}{l}\text { Vehicle route } \\
\text { planning } \\
\text { software and } \\
\text { other sources. }\end{array}$ & $\begin{array}{l}\text { Based on } \\
\text { architectural } \\
\text { projects and energy } \\
\text { certificates }\end{array}$ & $\begin{array}{l}\text { Authors } \\
\text { estimations } \\
\text { based on } \\
\text { Minister of } \\
\text { Construction } \\
\text { [95] }\end{array}$ \\
\hline & Fouquet et al., 2015 & - & Ecoinvent v3.01 & - & - & - & - & $\begin{array}{l}\text { Authors } \\
\text { estimations } \\
\text { based on previous } \\
\text { research [18] }\end{array}$ & $\begin{array}{l}\text { Authors } \\
\text { estimations }\end{array}$ & Comfie & \\
\hline & $\begin{array}{l}\text { Peuportier et al. } \\
2013\end{array}$ & - & $\begin{array}{l}\text { Ecoinvent. v1.1 } \\
\text { and further } \\
\text { versions. }\end{array}$ & - & - & $\begin{array}{l}\text { Authors } \\
\text { estimations }\end{array}$ & $\begin{array}{l}\text { Authors } \\
\text { estimations }\end{array}$ & $\begin{array}{l}\text { Authors } \\
\text { estimations }\end{array}$ & $\begin{array}{l}\text { Authors } \\
\text { estimations }\end{array}$ & Comfie & - \\
\hline \multirow{5}{*}{$\begin{array}{l}\text { 2. Non- } \\
\text { European } \\
\text { cases }\end{array}$} & $\begin{array}{l}\text { Babaizadeh et al., } \\
2015\end{array}$ & BEES model & $\begin{array}{l}\text { Ecoinvent } 3.0 \\
\text { BEES model }\end{array}$ & - & - & $\begin{array}{l}\text { Authors } \\
\text { estimations }\end{array}$ & $\begin{array}{l}\text { Authors } \\
\text { estimations }\end{array}$ & $\begin{array}{l}\text { Ecoinvent } 3.0 \\
\text { BUWAL } 250\end{array}$ & - & EnergyPlus 7.2 & - \\
\hline & Utama et al., 2012 & - & $\begin{array}{l}\text { Previous research } \\
{[96]}\end{array}$ & $\begin{array}{l}\text { Authors } \\
\text { estimations }\end{array}$ & $\begin{array}{l}\text { Authors } \\
\text { estimations }\end{array}$ & - & - & - & - & Authors estimations & - \\
\hline & Hanandeh, 2015 & - & $\begin{array}{l}\text { ELCD database } 2.0 \\
\text { and previous } \\
\text { research }[97,98]\end{array}$ & - & - & $\begin{array}{l}\text { Authors } \\
\text { estimations }\end{array}$ & $\begin{array}{l}\text { Authors } \\
\text { estimations }\end{array}$ & $\begin{array}{l}\text { Statistic } \\
\text { estimations }\end{array}$ & - & $\begin{array}{l}\text { Statistic estimations } \\
\text { and previous } \\
\text { research } \\
{[99]}\end{array}$ & - \\
\hline & Islam et al., 2014 & $\begin{array}{l}\text { BBQ (Builder's } \\
\text { bill of quantity) }\end{array}$ & $\begin{array}{l}\text { AusLCI database } \\
\text { Ecoinvent }\end{array}$ & - & $\begin{array}{l}\text { Authors } \\
\text { estimations }\end{array}$ & - & - & $\begin{array}{l}\text { Authors } \\
\text { estimations }\end{array}$ & $\begin{array}{l}\text { Authors } \\
\text { estimations }\end{array}$ & AccuRate software & - \\
\hline & $\begin{array}{l}\text { Oyarzo \& Peuportier, } \\
2014\end{array}$ & - & Ecoinvent v1.1 & - & - & - & - & $\begin{array}{l}\text { Authors } \\
\text { estimations }\end{array}$ & $\begin{array}{l}\text { Authors } \\
\text { estimations }\end{array}$ & $\begin{array}{l}\text { Comfie and } \\
\text { multizone approach }\end{array}$ & $\begin{array}{l}\text { Authors } \\
\text { estimations } \\
\text { based }\end{array}$ \\
\hline $\begin{array}{l}3 . \\
\text { European and } \\
\text { Non-European } \\
\text { cases }\end{array}$ & $\begin{array}{l}\text { Mosteiro-Romero } \\
\text { et al., } 2014\end{array}$ & - & Ecoinvent v2.2 & - & - & $\begin{array}{l}\text { Authors } \\
\text { estimations }\end{array}$ & $\begin{array}{l}\text { Authors } \\
\text { estimations }\end{array}$ & $\begin{array}{l}\text { Authors } \\
\text { estimations and } \\
\text { previous research } \\
{[100]}\end{array}$ & $\begin{array}{l}\text { Authors } \\
\text { estimations }\end{array}$ & $\begin{array}{l}\text { REM/DesignTM } \\
\text { Software }\end{array}$ & - \\
\hline
\end{tabular}




\subsubsection{Life cycle Impact Calculation (LCAI) and interpretation Phases}

Among the selected papers a wide variety of environmental impact indicators were identified. Different criteria in environmental impact indicators definitions were recognized. Some of them corresponded to global environmental priorities and some of them responded to regional environmental priorities. About Global priorities Table 1 shows EN 15978 [13] environmental indicators categories including: Environmental impact indicators, Resource Use and Waste categories and the output flow leaving the system. $75 \%$ of the selected papers analyzed focused on some of those Environmental Impact Indicators, GWP was mostly considered. Strictly speaking, Resource Use indicators and Waste categories indicators were not considered. About local priorities Hanandeh [32] defines the environmental impact indicators by focusing on the most significant environmental problems in Jordan: WD, CC, AP, HTP, and PMF. Islam et al. [44] define global -GHG and CED- and regional categories -water use and solid waste-.

On the other hand, several cases focus on $\mathrm{CO}_{2}$ emissions; one of the most significant environmental indicators in climate change mitigation. Moncaster \& Symon [101] recommend its calculation as a part of building design process. Rossi et al. [38], Iddon et al. [39], Houlihan et al. [43] calculated Carbon emissions Embodied and Operational-. Moreover, Rosselló-Batle et al. [60] focused on Embodied and Operational Energy. 


\subsection{Analysis of the life cycle stages and scenario definition}

Among the selected papers, it was detected that none of the cases included all modules. Reduction of stages and modules depended on the aim of the study. The major reductions were detected in use, demolition, and loads and benefits stages. In that respect, $30 \%$ of cases studies omitted two or more LCA stages. Utama et al. [34], compared direct and indirect emissions of building envelope materials on product, construction and maintenance modules. Houlihan et al. [43] calculated environmental impact of complete building considering production, maintenance and operational energy and water. Iddon et al. [39] compared four different construction scenarios for a typical house including production and operational energy use. Rossi et al. [38] compared embodied and operational consumption for a house in three different European locations by considering production, transport to site, operational energy and water use. Rosselló-Batle et al. [60] also compared embodied and operational energy demand in a Mediterranean climate by including production stage, maintenance - partially- and operational energy. Several omitted modules were justified by previous research.

Furthermore, more than $70 \%$ of papers included all LCA stages within system limits -production, construction, use and demolition-. Gervasio et al. [42] introduced an LCA application for early stages of design. The study is the most complete case; it included almost all modules, except A5, B1 y B7. Lewandowska et al. [36] became one of the most complete cases, by including: A2-A5, B1, B2, B3, B4, B6, B7, C1, C2 and C4. Islam et al. (2014) included: A1-A5, B2, B4, B6, C1 and C2. Dahlstrøm et al. [41] included: A1-A5, partially B2 (just for ventilation and heating systems), B6, B7, C1 and C3. Hanandeh [32] included all LCA stages: production, construction, operational stage -just B2, B6, B7- and recycling - just for aluminum windows and frames. Babaizadeh et al. [31] omitted A5, B1-B5 and B7.

\subsubsection{Analysis of the production stage}

Production stage was included in all the selected papers. It referred to the phase that data collection is more accessible than use or end of life phase. Table 3 demonstrates that it was the phase where generic database or other sources were mostly used.

\subsubsection{Analysis of the use stage}

Use stage was the most heterogeneous phase in terms of module assessment; none of the selected papers had totally considered this. Operational energy use was included in $95 \%$ of cases, while replacement, refurbishment and repair were hardly included. It was confirmed that the use stage had the most uncertainties in 
data collection, because of the lack of data bases, statistics or any other criteria that simplified the process. According to Table 3 data sources about the use stage were mostly based on the authors' estimations. 
Table 5.

Comparison of life cycle stages considered on reviewed papers.

\begin{tabular}{|c|c|c|c|c|c|c|c|c|c|c|c|c|c|c|c|c|c|c|}
\hline & & \multicolumn{16}{|c|}{ Building life cycle information } & \multirow{2}{*}{$\begin{array}{c}\begin{array}{c}\text { Suppletory Information beyond } \\
\text { Build. life cycle }\end{array} \\
\text { D } \\
\text { Benefits / Loads }\end{array}$} \\
\hline & & & $\begin{array}{l}\text { A1-A } \\
\text { oduct }\end{array}$ & & & $\begin{array}{l}\text {-A6 } \\
\text { truction } \\
\text { age }\end{array}$ & & & & $\begin{array}{l}\text { B1-B } \\
\text { Use sta }\end{array}$ & & & & & $\begin{array}{l}\text { C1-C } \\
\text { of lif }\end{array}$ & & & \\
\hline & 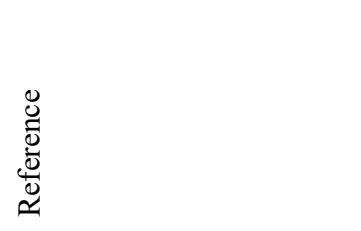 & 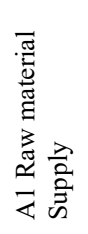 & 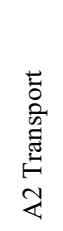 & 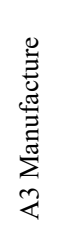 & 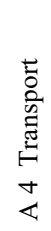 & 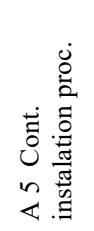 & $\begin{array}{l}0 \\
\stackrel{\infty}{D} \\
\bar{n}\end{array}$ & 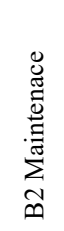 & 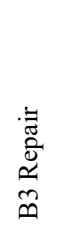 & 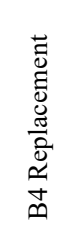 & 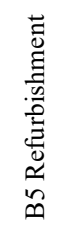 & 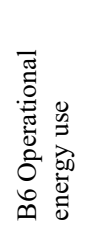 & 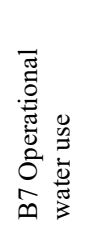 & 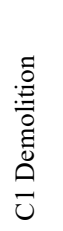 & 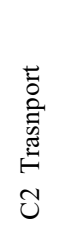 & 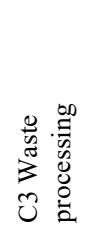 & 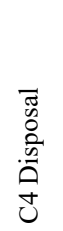 & 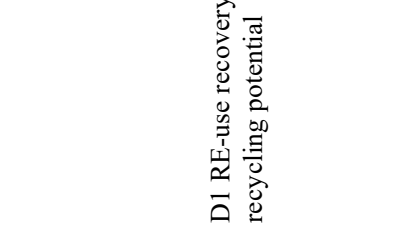 \\
\hline \multirow{14}{*}{$\begin{array}{l}1 . \\
\text { European } \\
\text { Cases }\end{array}$} & Houlihan et al., 2014 & $\mathrm{X}$ & $\mathrm{X}$ & $\mathrm{X}$ & - & - & - & - & - & $\mathrm{X}$ & - & $\mathrm{x}$ & - & - & - & - & - & - \\
\hline & Gervasio et al., 2014 & $\mathrm{X}$ & $\mathrm{X}$ & $\mathrm{X}$ & $\mathrm{X}$ & - & - & $\mathrm{X}$ & $\mathrm{x}$ & $\mathrm{X}$ & $\mathrm{X}$ & $\mathrm{X}$ & - & $\mathrm{X}$ & $\mathrm{X}$ & $\mathrm{X}$ & $\mathrm{X}$ & $\mathrm{X}$ \\
\hline & Dahlstrøm et al., 2012 & $\mathrm{X}$ & $\mathrm{X}$ & $\mathrm{X}$ & $\mathrm{X}$ & $\mathrm{X}$ & - & $\mathrm{X}$ & - & - & - & $\mathrm{X}$ & $\mathrm{X}$ & $\mathrm{X}$ & $X$ & $X$ & - & - \\
\hline & Motuzienè et al., 2016 & $\mathrm{X}$ & $\mathrm{X}$ & $\mathrm{X}$ & $\mathrm{X}$ & $\mathrm{X}$ & - & $\mathrm{X}$ & - & $\mathrm{X}$ & - & $\mathrm{X}$ & $\mathrm{X}$ & $\mathrm{X}$ & $\mathrm{X}$ & $\mathrm{X}$ & $\mathrm{X}$ & - \\
\hline & Proietti et al., 2013 & $\mathrm{X}$ & $\mathrm{X}$ & $\mathrm{X}$ & $\mathrm{X}$ & $\mathrm{X}$ & - & $\mathrm{X}$ & - & - & - & $\mathrm{x}$ & - & $\mathrm{X}$ & $\mathrm{X}$ & $\mathrm{X}$ & $\mathrm{X}$ & $(\mathrm{X})$ \\
\hline & Takano et al., 2015 & $\mathrm{X}$ & $\mathrm{X}$ & $\mathrm{X}$ & - & - & - & $\mathrm{X}$ & - & $\mathrm{X}$ & - & $\mathrm{x}$ & - & $\mathrm{X}$ & $\mathrm{X}$ & $\mathrm{X}$ & $\mathrm{X}$ & $\mathrm{X}$ \\
\hline & $\begin{array}{l}\text { Cuéllar-Franca \& Azapagic, } \\
2012\end{array}$ & $\mathrm{X}$ & $\mathrm{X}$ & $\mathrm{X}$ & $\mathrm{x}$ & $\mathrm{X}$ & - & $\mathrm{X}$ & - & $\mathrm{X}$ & - & $\mathrm{x}$ & $\mathrm{X}$ & $\mathrm{X}$ & $\mathrm{X}$ & $\mathrm{X}$ & $\mathrm{X}$ & $\mathrm{X}$ \\
\hline & Iddon et al., 2013 & $X$ & $X$ & $X$ & - & - & - & - & - & - & - & $X$ & - & - & - & - & - & - \\
\hline & Monteiro \& Freire, 2012 & $\mathrm{X}$ & $\mathrm{X}$ & $\mathrm{X}$ & $\mathrm{X}$ & $\mathrm{X}$ & - & $\mathrm{X}$ & - & $\mathrm{X}$ & - & $\mathrm{X}$ & - & - & - & - & - & - \\
\hline & Rosselló-Batle et al., 2015 & $\mathrm{X}$ & $\mathrm{X}$ & $\mathrm{X}$ & $\mathrm{X}$ & - & - & - & - & - & - & $\mathrm{X}$ & - & - & - & - & - & - \\
\hline & Lewandowska et al., 2013 & - & $\mathrm{X}$ & $\mathrm{X}$ & $\mathrm{X}$ & $\mathrm{X}$ & $\mathrm{X}$ & $\mathrm{X}$ & $\mathrm{X}$ & $\mathrm{X}$ & - & $\mathrm{X}$ & $\mathrm{X}$ & $\mathrm{X}$ & $\mathrm{X}$ & - & $\mathrm{X}$ & - \\
\hline & Rossi et al., 2015 & $\mathrm{X}$ & $\mathrm{X}$ & $\mathrm{X}$ & $\mathrm{x}$ & - & - & - & - & - & - & $\mathrm{X}$ & $\mathrm{X}$ & - & - & - & - & \\
\hline & Fouquet et al., 2015 & $\mathrm{X}$ & $\mathrm{X}$ & $\mathrm{X}$ & $\mathrm{X}$ & $\mathrm{X}$ & - & - & - & - & $\mathrm{X}$ & $\mathrm{X}$ & - & $\mathrm{X}$ & $\mathrm{X}$ & $\mathrm{X}$ & $\mathrm{X}$ & - \\
\hline & Peuportier et al., 2013 & $\mathrm{X}$ & $\mathrm{X}$ & $\mathrm{X}$ & $\mathrm{X}$ & $\mathrm{X}$ & - & $\mathrm{X}$ & - & - & - & $\mathrm{X}$ & $\mathrm{X}$ & $\mathrm{X}$ & $\mathrm{X}$ & - & $\mathrm{X}$ & - \\
\hline \multirow{5}{*}{$\begin{array}{l}2 . \\
\text { Non- } \\
\text { European } \\
\text { Cases }\end{array}$} & Babaizadeh et al., 2015 & $\mathrm{X}$ & $\mathrm{X}$ & $\mathrm{X}$ & $\mathrm{X}$ & - & - & - & - & - & - & $\mathrm{X}$ & - & $\mathrm{X}$ & $\mathrm{X}$ & $\mathrm{X}$ & $\mathrm{X}$ & $\mathrm{x}$ \\
\hline & Utama et al., 2012 & $\mathrm{X}$ & $\mathrm{X}$ & $\mathrm{X}$ & $\mathrm{X}$ & $\mathrm{x}$ & - & $\mathrm{X}$ & - & $\mathrm{X}$ & - & - & - & - & - & - & - & - \\
\hline & Hanandeh, 2015 & $\mathrm{X}$ & $\mathrm{X}$ & $\mathrm{X}$ & $\mathrm{X}$ & $\mathrm{X}$ & - & $\mathrm{X}$ & - & - & - & $\mathrm{X}$ & $\mathrm{X}$ & - & $\mathrm{X}$ & - & $\mathrm{X}$ & $\mathrm{X}$ \\
\hline & Islam et al., 2014 & $\mathrm{X}$ & $\mathrm{X}$ & $\mathrm{X}$ & $\mathrm{x}$ & $\mathrm{X}$ & - & $\mathrm{X}$ & - & - & - & $\mathrm{X}$ & - & $\mathrm{X}$ & $\mathrm{X}$ & - & - & - \\
\hline & Oyarzo \& Peuportier, 2014 & $\mathrm{X}$ & $\mathrm{X}$ & $\mathrm{X}$ & $\mathrm{X}$ & $\mathrm{X}$ & $\mathrm{X}$ & $\mathrm{X}$ & $\mathrm{X}$ & - & - & $\mathrm{X}$ & $\mathrm{X}$ & $\mathrm{X}$ & $\mathrm{X}$ & $\mathrm{X}$ & - & - \\
\hline $\begin{array}{l}\text { 3. European } \\
\text { and Non- } \\
\text { European } \\
\text { cases }\end{array}$ & Mosteiro-Romero et al., 2014 & $\mathrm{X}$ & $\mathrm{X}$ & $\mathrm{X}$ & $\mathrm{x}$ & $\mathrm{X}$ & - & $\mathrm{X}$ & - & $\mathrm{X}$ & - & $\mathrm{x}$ & - & $\mathrm{X}$ & $\mathrm{X}$ & $\mathrm{X}$ & $\mathrm{X}$ & $\mathrm{X}$ \\
\hline
\end{tabular}




\subsubsection{Analysis of the end of life stage}

End of life stage was omitted in $20 \%$ of the case studies, and several strategies were detected. Peuportier et al. [61], for instance, assumed landfill for demolition waste as a way for simplifying end of life stage definition. Fouquet et al. [62] defined two scenarios for a timber frame system, comparing incineration and landfill. Babaizadeh et al. [31] also described different scenarios for building materials: $75 \%$ of wood is assumed to be used for landfill, the rest $25 \%$ is incinerated, and aluminum and PVC is assumed to be recycled. On the other hand, Monteiro \& Freire [63], justify the end of life omissions by considering the limited importance of end-of-life stage impacts in South European single-family houses, demonstrated in Nemry et al. [104] .

\subsubsection{Analysis of the benefits and loads}

Benefits and loads were hardly included. Several cases included partial accounts. Hanandeh [32] for example considered benefits only for aluminum windows and frames. Other cases justified the omission. Dahlstrøm et al. [41] assumed the worst-case waste scenario, so no gains from reuse or recycling material were considered.

\section{DISCUSSION}

Reference literature $[9,29]$ assumes that simplifications to LCA referred to system boundary definition, data acquisition and results communication. Therefore, simplifications of the LCA method refer to reducing and optimizing those aspects. Based on that fact, from publications analyzed the following issues are discussed: strategies that search to reduce the amount of data and processes - Study of object boundary-, strategies that search to optimize life cycle stages and scenario definition -Life cycle stages and scenario definition-, and finally, strategies that search to simplify impact calculation and result communication -Impact calculation and results communication-.

\subsection{Study of object boundary}

The study of object definition involves setting limits to real building. Detected simplification strategies are mostly focused on reducing the amount of data, optimizing data collection and simplifying LCA. According to EN 15978 [13] system boundaries and functional unit definition do not only refer to complete building application, it also allows for partial assessment of buildings, e.g. building envelope, windows, roofs, etc. So, that reduction is considered as a simplification strategy. Among papers reviewed, $35 \%$ of partial or reference 
area applications are detected. Babaizadeh et al. [31] focus the study on shading assessment. Gervasio et al. [42], for instance, develop a macro-component approach. Dahlstrøm et al. [41] reduce the functional unit to a $1 \mathrm{~m}^{2}$ of useable floor area.

Papers analyzed show growing interest in using BIM for developing the physical model of the building $[3,39,43] .[3]$

Although, Houlihan et al. [43] recognize the existence of several limitations in calculation of some building components, it demonstrates that BIM is a helpful format for quantifying material inputs. The lack of data sources and the complexity in modelling building processes -such as transport, maintenance, repair, refurbishments, demolition, waste treatment or recycling-, is simplified in most cases as estimations based on previous research or regional data sources.

\subsection{Life cycle stages and scenario definition}

The review demonstrates that life cycle stages definitions also play an important role to simplified LCA for single-family houses. Among papers analyzed, no complete modules-A1 to D- assessment is detected. So according to the EeB Guide [14] type classification, most of the cases correspond to Simplified LCA application type. The review also demonstrates the relevance of product stages and operational energy use module in LCA for single-family houses. Product stage (A1 to A3) is included in all cases and Operational energy consumption (OE) is also mostly considered. Cuellár-Franca \& Azapagic [37] show that it represents about 90\% of GWP.

Regarding case studies, reduction and simplification in life-cycle stages, these are related to the scope and objectives of each case. According to Houlihan et al. [43], Iddon et al. [39], Rossi et al. [38], Rosselló-Batle et al. [60], if the assessment is focused on comparing embodied and operational impacts, life-cycle stages definition is centered on product stages and energy consumption during use module. In other cases [3,31$33,35,36,40,41,44,45,62,61,105]$, if the assessment is focused on environmental impact during the life-cycle; product, construction, use and demolition are mostly included, while loads and benefits are hardly considered. It is also detected in Gervasio et al. [42] that early stages of design assessments are focused on products, construction, use -partially-, and demolition stages. Utama et al. [34] center on building material performance; product, construction and use are partially considered.

Furthermore life cycle stages and scenario definitions are heterogeneous in all case studies. Product stages scenario is mostly recognized to be supported by generic databases as shown Table 4. However, EN 
15798 [13] assumes the use of EPD as a simplification to product stage scenario definition. Among papers reviewed no EPD applications are detected. Use stage scenario definition is directly related to construction materials, climate, technology, and other regional characteristics. Simplification strategies aim to facilitate access to uncertain information. In this context, most of the papers considered are based on the authors' estimations or previous research as shown in Table 4. Moreover, the use of Dynamic Thermal Simulations tool to help on Operational energy use scenarios definition is widely recognized. End-of-life stage was one of the most heterogeneous stages. Detected simplification strategies assume in several cases landfill, justified omission, or incineration, as an alternative for a waste treatment scenario. In spite of that fact the European context goals by 2020 aim to reuse, recycle or recovery of material of at least $70 \%$ of non-hazardous construction and demolition waste [106].

\subsection{Impact calculation and results communication}

Results demonstrate that depending on Impact calculation methods, final results can differ. Moreover, calculation methods are defined based on selected environmental impact indicator. Monteiro \& Freire [63] demonstrate the influence of Impact calculation methods in LCA results. The study, that compares three of the most used calculation methods -CML, Ecoindicator' 99 and CED-, demonstrates that by using these methods inconsistent results can be obtained for HTP, ET and PODP. So, the study suggests that LCA for single-family houses ought to include multiple environmental impacts and ought not to include toxicity categories.

Several cases have cut down the number of impact indicators by considering different criteria as: regional representativeness, global impact, embodied vs operational impact, renewable vs non-renewable energy consumption. From the analysis of the selected papers it is demonstrated that this simplification strategy can reduce the amount of data and complexity in LCA application, without altering result comparability. According to the selected papers GWP is the most significant environmental indicator; globally recognized as one of the most significant indicators for climate change mitigation strategies, and also takes part of the EN 15978 [13] required environmental indicators. Almost $20 \%$ of case studies $[33,35,62,105]$ that considered complete building as the functional unit have calculated and included GWP results in the paper. Those cases were compared in Fig. $\mathbf{2}$, in spite of the fact that methodological differences between them are recognized.

Fig. 2 summarized the GWP ratio (kg. eq. $\mathrm{CO}_{2} / \mathrm{m}^{2} / \mathrm{yr}$.) obtained from selected cases studies. According to Fig. 2 results obtained are heterogeneous. Only two cases $[33,105]$ considered similar service lifetime (50 years), similar life cycle stages and the same impact calculation method (CML 2001 method). For these cases, the GWP 
ratio ranges from 23 to $105 \mathrm{~kg}$. eq. $\mathrm{CO}_{2} / \mathrm{m}^{2} / \mathrm{yr}$. and the media value for typical or reference scenario was $57 \mathrm{~kg}$.

eq. $\mathrm{CO}_{2} / \mathrm{m}^{2} / \mathrm{yr}$.

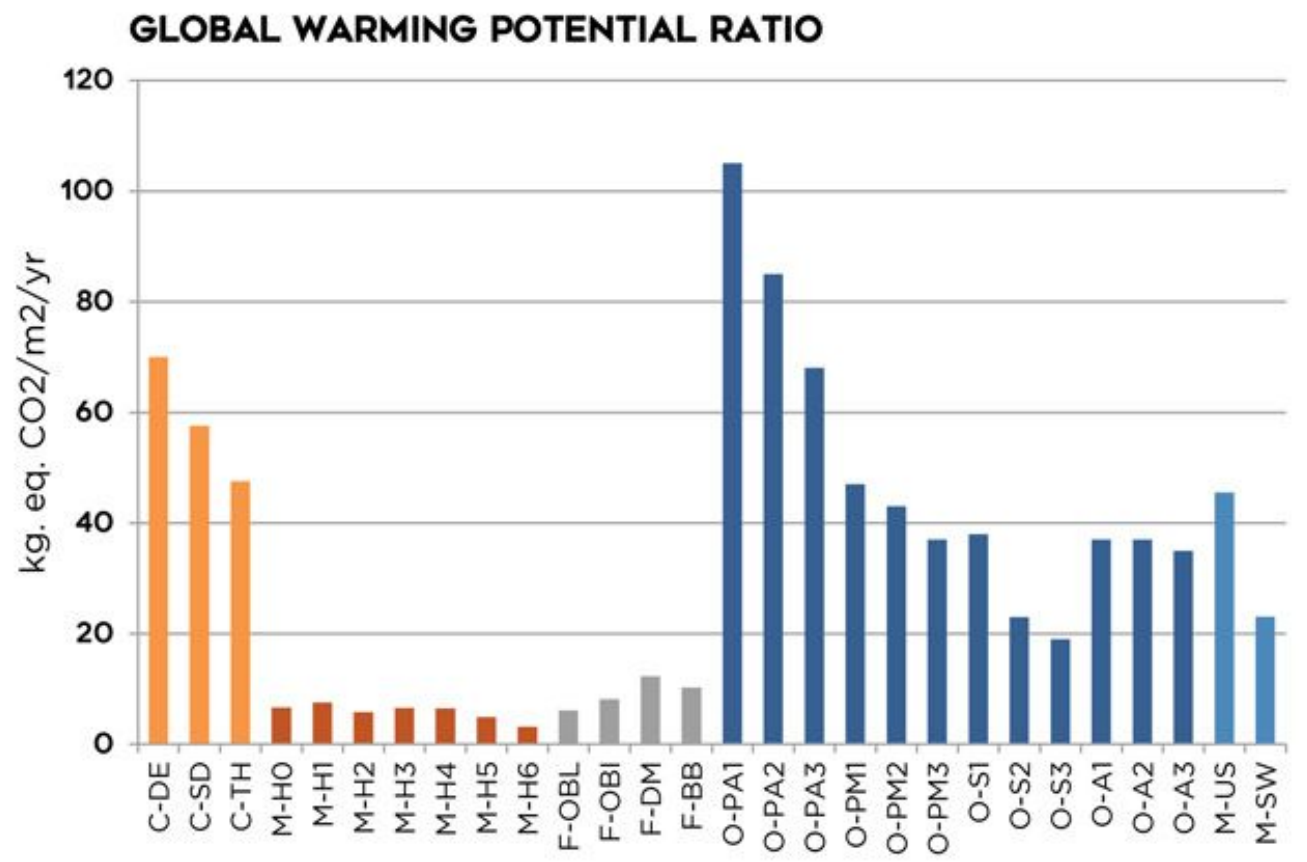

\section{CASE STUDIES}

= Cuéllar-Franca \& Azapagic, 2012

= Monteiro \& Freire, 2012

=Fouquet et al., 2015

- Oyarzo \& Peuportier, 2014

- Mosteiro-Romero et al., 2014

Fig. 2. Comparison of GWP ratio (kg. eq. $\mathrm{CO}_{2} / \mathrm{m}^{2} / \mathrm{yr}$.) from selected case studies [Selected case studies: Cuéllar-Franca \& Azapagic: C-DE, detached house; C-SD, semi-detached house; C-TH terraced house; Monteiro \& Freire: M-H0, reference house with double hollow brick masonry; M-H1 reference house with double facing and hollow brick masonry; M-H2, reference house with light weight concrete block masonry; M-H3, reference house with thermal concrete blocks masonry; M$\mathrm{H} 4$, reference house with autoclaved aerated concrete block masonry; M-H5, reference house with hollow brick masonry \& ext. wood cladding; M-H6, reference house with wood frame and cladding; Fouquet et al.: F-OBL, reference house considering timber frame landfill scenario; F-OBI, reference house considering timber frame incineration scenario; F-DM, reference house with concrete blocks cavity walls; F-BB, reference house with cast concrete walls; Oyarzo \& Peuportier: OPA1, reference house in Punta Arenas; O-PA2, regulatory house in Punta Arenas; O-PA3, improved house in Punta Arenas; O-PM1, reference house in Puerto Montt; O-PM2, regulatory house in Puerto Montt; O-PM3, improved house in Puerto Montt; O-S1, reference house in Santiago; O-S2, regulatory house in Santiago; O-S3, improved house in Santiago; MosteiroRomero et al.: M-US, LEED-H certified house in US; M-SW, Minergie-P certified house in Switzerland].

Results communications play a key role to organize and compare calculated environmental impact. Almost half of the selected papers analyzed [3,35,38-41,45] organize results according to building parts -e.g. external walls, internal walls, windows, roof, shadings, etc.-. Several cases $[31,34,43,60,105]$ organize results by classification of construction materials. Cuéllar-Franca \& Azapagic [105], Fouquet et al. [62], Monteiro \& Freire [63], Lewandowska et al. [36], Proietti et al. [45], Oyarzo \& Peuportier [33], Peuportier et al. [61], Mosteiro- 
Romero et al. [35] organize results by LCA stages, adapting them to the particular case. Islam et al. [44] compare different building type impact and Hanandeh [32] develop an environmental impact comparison. Other cases [40,42] present results by stage and modules criteria described in EN 15798 [13].Takano et al. [40] for example, combine LCA stages and building part organization, so it allows for partial comparison or complete LCA application.

\section{CONCLUSIONS}

The papers analyzed show growing interest in LCA for single-family houses and the trend towards simplifying environmental assessment based on the LCA method. Results show that the focus on simplification is set on system boundary definition - including model definition and life cycle stages definition-, on scenarios definition and on results communication. The review confirms that simplifications to real buildings are necessary during LCA application, however it is necessary to know their limits and how it affects reliability, transparency and comparability of the results.

Selected papers, demonstrate the influence of EN 15978 [13] in the LCA for single-family houses during the last five years and its relevance as a methodological guidance for them. Although it is also demonstrated that the global reference on LCA for single-family houses are ISO standards [20,21], considered mostly in European and non-European cases. Furthermore, each of the selected papers analyzed proposes different application of the LCA method. Results show the usefulness of the LCA method to assess environmental impact during the lifecycle of the building or a part of it, to assess environmental impact during the early stages of design, and to compare embodied and operational impact. It is also demonstrated that simplification strategies can help on optimizing input account, reducing the amount of data, reducing the complexity of process assessment and impact assessment calculation.

The review confirms the existence of several simplification strategies as follow: optimization of data collection process, reduction of the functional unit, limitation of the study to relevant stages and modules, simplification of the scenario definition, use of databases or other generic data sources, use of calculation methods, and reduction of environmental indicators. It is demonstrated that simplification strategies refer in all cases to the scope and objectives of the study. On the other hand, due to the heterogeneity of simplification strategies it is not possible to ensure the comparability of the results. 
Finally, several recommendations and challenges for simplification strategies for single-family houses LCA can be concluded:

- $\quad$ Regarding the difficulties on data collection, it is needed to develop common criteria to define several processes as construction, transports, use, maintenance, replacements, repair, refurbishments and end-of-life processes according to regional characteristics, which can improve and provide guarantees to compare results.

- $\quad$ The use of EPD is still not verified in single-family houses LCA, in spite of being considered as a strategy to simplify product stage. This highlights the need to continue developing EPD for construction products.

About data quantification, the review demonstrates the growing interest in including BIM in LCA for single-family houses. Almost $15 \%$ of papers reviewed have recommended or used BIM models during LCA application. Further study would be needed to improve their complementarity.

- $\quad$ Regarding results communication, it is evident that EN 15978 [13] has improved LCA results communication. However, it is needed to go further to establish common criteria to compare results of similar building typologies and to simplify comparison of partial applications of LCA. Results can be presented by component or system that composes the building, -e.g. building envelope, windows, roof, and structure-. This would allow the comparison of environmental impact organized by life cycle stages, of components or systems of similar case studies.

Therefore, this paper demonstrates the need to further develop simplification strategies that do not alter results representativeness and also allow their comparability. It is also needed to extend the application of LCA in buildings -especially in single-family houses-, as well as to expand the use of environmental criteria in the building sector. 


\section{REFERENCES}

[1] United Nations Conference, Adoption of the Paris agreement, Paris, 2015.

[2] International Energy Agency, CO2 Emissions from Fuel Combustion. Beyond 2020 Online Database., Paris, 2012.

[3] V. Motuzienè, A. Rogoža, V. Lapinskienè, T. Vilutienė, Construction solutions for energy efficient single-family house based on its life cycle multi-criteria analysis: a case study, J. Clean. Prod. 112 (2016) 532-541. doi:10.1016/j.jclepro.2015.08.103.

[4] C. Petersdorff, T. Boermans, J. Harnisch, Mitigation of CO2 emissions from the EU-15 building stock: beyond the EU Directive on the Energy Performance of Buildings., Environ. Sci. Pollut. Res. Int. 13 (2006) 350-358. doi:10.1007/BF03039568.

[5] M. Weissenberger, W. Jensch, W. Lang, The convergence of life cycle assessment and nearly zeroenergy buildings: The case of Germany, Energy Build. 76 (2014) 551-557. doi:10.1016/j.enbuild.2014.03.028.

[6] L.F. Cabeza, L. Rincón, V. Vilariño, G. Pérez, A. Castell, Life cycle assessment (LCA) and life cycle energy analysis (LCEA) of buildings and the building sector: A review, Renew. Sustain. Energy Rev. 29 (2014) 394-416. doi:10.1016/j.rser.2013.08.037.

[7] M. Lotteau, P. Loubet, M. Pousse, E. Dufrasnes, G. Sonnemann, Critical review of life cycle assessment (LCA) for the built environment at the neighborhood scale, Build. Environ. 93 (2015) 165-178. doi:10.1016/j.buildenv.2015.06.029.

[8] M. Buyle, J. Braet, A. Audenaert, Life cycle assessment in the construction sector: A review, Renew. Sustain. Energy Rev. 26 (2013) 379-388. doi:10.1016/j.rser.2013.05.001.

[9] T. Malmqvist, M. Glaumann, S. Scarpellini, I. Zabalza, A. Aranda, E. Llera, et al., Life cycle assessment in buildings: The ENSLIC simplified method and guidelines, Energy. 36 (2011) 1900-1907. doi:10.1016/j.energy.2010.03.026.

[10] V. John, Derivation Of Reliable Simplification Strategies For The Comparative Lca Of Individual And “Typical“ Newly Built Swiss Apartment Buildings, ETH ZURICH, 2012. doi:dx.doi.org/10.3929/ethz-a007607252.

[11] J. Basbagill, F. Flager, M. Lepech, M. Fischer, Application of life-cycle assessment to early stage building design for reduced embodied environmental impacts, Build. Environ. 60 (2013) 81-92. doi:10.1016/j.buildenv.2012.11.009. 
[12] EN 15804, BS EN 15804:2012 - Standards Publication Sustainability of construction works Environmental product declarations - Core rules for the product category of construction products, Int. Stand. (2014) 70.

[13] EN 15978, BS EN 15978:2011 - Sustainability of construction works - Assessment of environmental performance of buildings - Calculation method, Int. Stand. (2011).

[14] EeB Guide Project, Operational Guidance for Life Cycle Assessment Studies of the Energy Efficient Buildings Initiative, (2012). http://www.eebguide.eu/.

[15] C. Lamnatou, D. Chemisana, R. Mateus, M.G. Almeida, S.M. Silva, Review and perspectives on Life Cycle Analysis of solar technologies with emphasis on building-integrated solar thermal systems, Renew. Energy. 75 (2015) 833-846. doi:10.1016/j.renene.2014.09.057.

[16] D. Kellenberger, H.J. Althaus, Relevance of simplifications in LCA of building components, Build. Environ. 44 (2009) 818-825. doi:10.1016/j.buildenv.2008.06.002.

[17] I. Zabalza Bribián, A. Aranda Usón, S. Scarpellini, Life cycle assessment in buildings: State-of-the-art and simplified LCA methodology as a complement for building certification, Build. Environ. 44 (2009) 2510-2520. doi:10.1016/j.buildenv.2009.05.001.

[18] S. Lasvaux, Etude d'un modele simplifie pour l'analyse de cycle de vie des batiments, Ecole Nationale Superieure des Mines de Paris, 2010. https://pastel.archives-ouvertes.fr/pastel-00712043.

[19] A. Takano, A. Hafner, L. Linkosalmi, S. Ott, M. Hughes, S. Winter, Life cycle assessment of wood construction according to the normative standards, Eur. J. Wood Wood Prod. 73 (2015) 299-312. doi:10.1007/s00107-015-0890-4.

[20] ISO, ISO 14040: Environmental management — Life Cycle Assessment — Principles and Framework, Environ. Manage. 3 (2006) 28. doi:10.1002/jtr.

[21] ISO, ISO 14044:2006, ISO. (2006). https://www.iso.org/obp/ui/\#iso:std:iso:14044:ed-1:v1:en.

[22] A. Passer, H. Kreiner, P. Maydl, Assessment of the environmental performance of buildings: A critical evaluation of the influence of technical building equipment on residential buildings, Int. J. Life Cycle Assess. 17 (2012) 1116-1130. doi:10.1007/s11367-012-0435-6.

[23] REGENER Project, REGENER Project, (n.d.). http://wwwcep.ensmp.fr/francais/themes/cycle/pdf/cib_regener.pdf.

[24] IEA, ANNEX 31, (n.d.). http://www.iisbe.org/annex31/index.html.

[25] European Commission, PRESCO Project, (n.d.). http://relay.iclei- 
europe.org/fileadmin/user_upload/relay/uploads/Kornadt.pdf.

[26] European Commission, IMPRO-Building, (n.d.). http://ftp.jrc.es/EURdoc/JRC46667.pdf.

[27] ENSLIC, (n.d.). http://circe.cps.unizar.es/enslic/texto/home.html.

[28] LORE-LCA, (n.d.). http://www.sintef.no/projectweb/lore-lca/.

[29] UNE 150041:1998 EX, Análisis de ciclo de vida simplificado, AENOR Stand. (1998).

[30] P. Karami, N. Al-Ayish, K. Gudmundsson, A comparative study of the environmental impact of Swedish residential buildings with vacuum insulation panels, Energy Build. 109 (2015) 183-194. doi:10.1016/j.enbuild.2015.10.031.

[31] H. Babaizadeh, N. Haghighi, S. Asadi, R. Broun, D. Riley, Life cycle assessment of exterior window shadings in residential buildings in different climate zones, Build. Environ. 90 (2015) 168-177. doi:10.1016/j.buildenv.2015.03.038.

[32] A. El Hanandeh, Environmental assessment of popular single-family house construction alternatives in Jordan, Build. Environ. 92 (2015) 192-199. doi:10.1016/j.buildenv.2015.04.032.

[33] J. Oyarzo, B. Peuportier, Life cycle assessment model applied to housing in Chile, J. Clean. Prod. 69 (2014) 109-116. doi:10.1016/j.jclepro.2014.01.090.

[34] N.A. Utama, B.C. Mclellan, S.H. Gheewala, K.N. Ishihara, Embodied impacts of traditional clay versus modern concrete houses in a tropical regime, Build. Environ. 57 (2012) 362-369. doi:10.1016/j.buildenv.2012.06.006.

[35] M. Mosteiro-Romero, U. Krogmann, H. Wallbaum, Y. Ostermeyer, J.S. Senick, C.J. Andrews, Relative importance of electricity sources and construction practices in residential buildings: A Swiss-US comparison of energy related life-cycle impacts, Energy Build. 68 (2014) 620-631. doi:10.1016/j.enbuild.2013.09.046.

[36] A. Lewandowska, A. Noskowiak, G. Pajchrowski, Comparative life cycle assessment of passive and traditional residential buildings' use with a special focus on energy-related aspects, Energy Build. 67 (2013) 635-646. doi:10.1016/j.enbuild.2013.09.002.

[37] R.M. Cuéllar-Franca, A. Azapagic, Environmental impacts of the UK residential sector: Life cycle assessment of houses, Build. Environ. 54 (2012) 86-99. doi:10.1016/j.buildenv.2012.02.005.

[38] B. Rossi, A.F. Marique, S. Reiter, Life-cycle assessment of residential buildings in three different European locations, case study, Build. Environ. 51 (2012) 402-407. doi:10.1016/j.buildenv.2011.11.002.

[39] C.R. Iddon, S.K. Firth, Embodied and operational energy for new-build housing: A case study of 
construction methods in the UK, Energy Build. 67 (2013) 479-488. doi:10.1016/j.enbuild.2013.08.041.

[40] A. Takano, S.K. Pal, M. Kuittinen, K. Alanne, M. Hughes, S. Winter, The effect of material selection on life cycle energy balance: A case study on a hypothetical building model in Finland, Build. Environ. 89 (2015) 192-202. doi:10.1016/j.buildenv.2015.03.001.

[41] O. Dahlstrøm, K. Sørnes, S.T. Eriksen, E.G. Hertwich, Life cycle assessment of a single-family residence built to either conventional- or passive house standard, Energy Build. 54 (2012) 470-479. doi:10.1016/j.enbuild.2012.07.029.

[42] H. Gervasio, P. Santos, R. Martins, L. Simoes da Silva, A macro-component approach for the assessment of building sustainability in early stages of design, Build. Environ. 73 (2014) 256-270. doi:10.1016/j.buildenv.2013.12.015.

[43] A. Houlihan Wiberg, L. Georges, T.H. Dokka, M. Haase, B. Time, A.G. Lien, et al., A net zero emission concept analysis of a single-family house, Energy Build. 74 (2014) 101-110. doi:10.1016/j.enbuild.2014.01.037.

[44] H. Islam, M. Jollands, S. Setunge, I. Ahmed, N. Haque, Life Cycle Assessment and Life Cycle Cost Implications of Wall Assemblages Designs, Energy Build. 84 (2014) 33-45. doi:10.1016/j.enbuild.2014.07.041.

[45] S. Proietti, P. Sdringola, U. Desideri, F. Zepparelli, F. Masciarelli, F. Castellani, Life Cycle Assessment of a passive house in a seismic temperate zone, Energy Build. 64 (2013) 463-472. doi:10.1016/j.enbuild.2013.05.013.

[46] Spon, Spon's mechanical \& electrical services price book, 42nd ed. United Kingdom: Spon Press, 2011.

[47] G. Verbeeck, H. Hens, Life cycle inventory of buildings: A contribution analysis, Build. Environ. 45 (2010) 964-967. doi:10.1016/j.buildenv.2009.10.003.

[48] A. Takano, S. Winter, M. Hughes, L. Linkosalmi, Comparison of life cycle assessment databases: A case study on building assessment, Build. Environ. 79 (2014) 20-30. doi:10.1016/j.buildenv.2014.04.025.

[49] B. Berge, The ecology of building materials, Great Britain: Architectural Press, 2001.

[50] J. Anderson, D. Shiers, M. Sinclair, The green guide to specification, Blackwell, Oxford, UK, 2002.

[51] DEFRA, Department for Environment Food and Rural Affairs, Sustainable consumption and production: domestic water consumption, (2010). http://webarchive.nationalarchives.gov.uk/20110223093550/ http://defra.gov.uk/sustainable/government/progress/national/16.

[52] J. Dewulf, G. Van Der Vorst, N. Versele, A. Janssens, J. Van Henderson, Comparison of running costs 
for different heating options in hard to treat flats., UK Energy Sav. Trust Build. Res. Establ. (2004).

[53] J. Prime, H. Addison, Estimates of heat use in the UK. London: Department of Energy and Climate Change, 2009.

[54] O. Ortiz, C. Bonnet, J.C. Bruno, F. Castells, Sustainability based on LCM of residential dwellings: A case study in Catalonia, Spain, Build. Environ. 44 (2009) 584-594. doi:10.1016/j.buildenv.2008.05.004.

[55] J. Utley, L. Shorrock, Domestic energy fact file, London Build. Res. Establ. (2008).

[56] DeWalt, Power Tools, 2011. (n.d.). http://www.dewalt.no/powertools/.

[57] SINTEF Byggforsk, Byggforskserien 474.533 - Byggfukt, Uttørking og forebyggende tiltak, 74 (2006).

[58] A. Horn, Dantherm air handling AS, (2011).

[59] F-Tech, Dry building, (2011). http://urn.kb.se/resolve?urn=urn:nbn:no:ntnu:diva-13635.

[60] B. Rosselló-Batle, C. Ribas, A. Moià-Pol, V. Martínez-Moll, An assessment of the relationship between embodied and thermal energy demands in dwellings in a Mediterranean climate, Energy Build. 109 (2015) 230-244. doi:10.1016/j.enbuild.2015.10.007.

[61] B. Peuportier, S. Thiers, A. Guiavarch, Eco-design of buildings using thermal simulation and life cycle assessment, J. Clean. Prod. 39 (2013) 73-78. doi:10.1016/j.jclepro.2012.08.041.

[62] M. Fouquet, A. Levasseur, M. Margni, A. Lebert, S. Lasvaux, B. Souyri, et al., Methodological challenges and developments in LCA of low energy buildings: Application to biogenic carbon and global warming assessment, Build. Environ. 90 (2015) 51-59. doi:10.1016/j.buildenv.2015.03.022.

[63] H. Monteiro, F. Freire, Life-cycle assessment of a house with alternative exterior walls: Comparison of three impact assessment methods, Energy Build. 47 (2012) 572-583. doi:10.1016/j.enbuild.2011.12.032.

[64] NS:3701, Criteria for passive houses and low energy buildings(non-residential buildings), Stand. Norge. (2012).

[65] NS:3700, Criteria for passive houses and low energy houses(residential buildings), 2010, Stand. Norge. (2010).

[66] NS:3031, Calculation of energy performance of buildings,methods and data, Stand. Norge. (2007).

[67] SIMIEN, SIMulation of Indoor climate and ENergy Use, Resource Action Programme. Byggerne Program, Skollenborg, n.d. www.wrap.org.uk/construction/case_studies/recycling_demolition.html.

[68] K. Sørnes, Heating and ventilation of a highly energy efficient residential buildings: environmental assessment of technology alternatives, Master Thesis, NTNU, 2011.

[69] SSB KOSTRA, Kommunal vannforsyning, (n.d.) 2009-2010. http://www.ssb.no/ emner/01/04/20/vann 
kostra/.

[70] SENSE, Systems Sustainability Evaluation of Solar Energy, Recycling of Production Waste, (2002). http://www.sense-eu.net/.

[71] SENSE, Systems Sustainability Evaluation of Solar Energy, LCA Analysis, (2008). http://www.senseeu.net/.

[72] O.F. Kofoworola, S.H. Gheewala, Life cycle energy assessment of a typical office building in Thailand, Energy Build. 41 (2009) 1076-1083. doi:10.1016/j.enbuild.2009.06.002.

[73] K. Adalberth, Energy use during the life cycle of single-unit dwellings: Examples, Build. Environ. 32 (1997) 321-329. doi:10.1016/S0360-1323(96)00069-8.

[74] G.A. Blengini, T. Di Carlo, The changing role of life cycle phases, subsystems and materials in the 5LCA6 of low energy buildings, Energy Build. 42 (2010) 869-880. doi:10.1016/j.enbuild.2009.12.009.

[75] T.. Chen, J. Burnett, C.. Chau, Analysis of embodied energy use in the residential building of Hong Kong, Energy. 26 (2001) 323-340. doi:10.1016/S0360-5442(01)00006-8.

[76] S. Citherlet, T. Defaux, Energy and environmental comparison of three variants of a family house during its whole life span, Build. Environ. 42 (2007) 591-598. doi:10.1016/j.buildenv.2005.09.025.

[77] M.Z. Hauschild, Assessing environmental impacts in a life-cycle perspective., Environ. Sci. Technol. 39 (2005) 81A-88A. doi:10.1021/es053190s.

[78] C. Scheuer, G.A. Keoleian, P. Reppe, Life cycle energy and environmental performance of a new university building: modeling challenges and design implications, Energy Build. 35 (2003) 1049-1064. doi:10.1016/s0378-7788(03)00066-5.

[79] L. Gustavsson, A. Joelsson, Life cycle primary energy analysis of residential buildings, Energy Build. 42 (2010) 210-220. doi:10.1016/j.enbuild.2009.08.017.

[80] M. Hauschild, J. Jeswiet, L. Alting, From Life Cycle Assessment to sustainable production: Status and perspectives, Cirp Ann. Technol. 54 (2005) 535-554. doi:10.1016/S0007-8506(07)60017-1.

[81] A. Mahdavi, E.M. Doppelbauer, A performance comparison of passive and low-energy buildings, Energy Build. 42 (2010) 1314-1319. doi:10.1016/j.enbuild.2010.02.025.

[82] G.A. Blengini, Life cycle of buildings, demolition and recycling potential: A case study in Turin, Italy, Build. Environ. 44 (2009) 319-330. doi:10.1016/j.buildenv.2008.03.007.

[83] C. Koroneos, G. Kottas, Energy consumption modeling analysis and environmental impact assessment of model house in Thessaloniki-Greece, Build. Environ. 42 (2007) 122-138. 
doi:10.1016/j.buildenv.2005.08.009.

[84] B. Polster, B. Peuportier, I. Blanc Sommereux, P. Diaz Pedregal, C. Gobin, E. Durand, Evaluation of the environmental quality of buildings towards a more environmentally conscious design, Sol. Energy. 57 (1996) 219-230. doi:10.1016/S0038-092X(96)00071-0.

[85] C. Thormark, A low energy building in a life cycle - its embodied energy, energy need for operation and recycling potential, Build. Environ. 37 (2002) 429-435. doi:10.1016/S0360-1323(01)00033-6.

[86] FIE, Finnish energy industries (Energiateollisuus ry). District heating in Finland 2013, (2014). http://energia.fi/en/statistics-and-publications/district-heating-statistics/district-heating.

[87] M. Brinkley, The housebuilder's bible, 7th ed. UK: Ovolo Publishing, 2008.

[88] B\&Q, B\&Q, (2011).www.diy.com/diy/jsp/?_requestid?32957.

[89] T. Barker, K. Jenkins, The domestic energy sub-model in MDM-E3, 2007.

[90] ISO, ISO 13790: 2008 Energy performance of buildings-Calculation of energy use for space heating and cooling, Order A J. Theory Ordered Sets Its Appl. 2008 (2008) 3190-200. doi:10.3168/jds.2007-0930.

[91] SAP Protocol, DECC, The Government's Standard Assessment Procedure for Energy Rating of Dwellings 2009 version 9.9, Crown, 2011.

[92] R. Frischknecht, N. Jungbluth, H.J. Althaus, G. Doka, R. Dones, H. R., et al., Implementation of life cycle impact assessment methods: data v2.0, 2007.

[93] M. Spielmann, R. Dones, C. Bauer, M. Tuchschmid, Life cycle inventories of transport services, 2007.

[94] D. Kellenberger, H.J. Althaus, N. Jungbluth, T. Künniger, M. Lehmann, P. Thalmann, Life cycle inventories of building products, Final report ecoinvent Data v2.0 No.7, Dübendorf, 2007.

[95] Minister of Construction, The regulation of the Minister of Construction as of 14 January 2002 relating to determination of mean water consumption standards, J. Laws. 8 (n.d.).

[96] A. Widiyanto, S. Kato, N. Maruyama, A. Nishimura, S. Sampattagul, Environmental impacts evaluation of electricity grid mix systems in four selected countries using a life cycle assessment point of view, in: 2003 EcoDesign 3rd Int. Symp. Environ. Conscious Des. Inverse Manuf., 2003: pp. 26-33. doi:10.1109/ECODIM.2003.1322633.

[97] University of Tennessee Center for Clean Products, Limestone quarrying and processing: a life-cycle inventory, Knoxville, TN 37996-4134: Natural Stone Council, 2008.

[98] M. Marceau, M. Nisbet, M. Van Geem, Life cycle inventory of Portland cement manufacture, 2006.

[99] Johansson E, D. Ouahrani, Editors., Climate conscious architecture and urban design in Jordan e towards 
energy efficient buildings and improved urban microclimate, n.d.

[100] B. A, E. M, G.. Lehmann, kologische Nachhaltigkeit imWohnungsbau; eine Bewertung von Erneuerungsstrategie, Muttenz: Fachhochschule beider Basel, 2000.

[101] A.M. Moncaster, K.E. Symons, A method and tool for "cradle to grave" embodied carbon and energy impacts of UK buildings in compliance with the new TC350 standards, Energy Build. 66 (2013) 514523. doi:10.1016/j.enbuild.2013.07.046.

[102] A. Takano, M. Hughes, S. Winter, A multidisciplinary approach to sustainable building material selection: A case study in a Finnish context, Build. Environ. 82 (2014) 526-535. doi:10.1016/j.buildenv.2014.09.026.

[103] R. Frischknecht, R. Steiner, N. Jungbluth, The ecological scarcity method e ecofactors 2006. A method for impact assessment in LCA., Bern: Federal Office for the Environment., 2009.

[104] F. Nemry, A. Uihlein, C.M. Colodel, B. Wittstock, A. Braune, C. Wetzel, et al., Environmental Improvement Potentials of Residential Buildings (IMPRO-Building), 2008. doi:10.2791/38942.

[105] R.M. Cuéllar-Franca, A. Azapagic, Life cycle cost analysis of the UK housing stock, Int. J. Life Cycle Assess. 19 (2014) 174-193. doi:10.1007/s11367-013-0610-4.

[106] European Commission, Directive 2008/98/EC of the European Parliament and of the Council on waste and repealing certain Directives, 2008. 
Highlights:

- Review of recent single-family houses LCA, focused on simplification strategies.

- Simplification mainly focused on system boundary and results communication.

- The most considered environmental impact indicator was GWP.

- Future studies need simplification strategies that allow results comparability. 